PALEO

Revue d'archéologie préhistorique

$14 \mid 2002$

Varia

\title{
La dent humaine de Pradayrol (Caniac-du-Causse, Lot) dans son contexte stratigraphique et paléontologique
}

The human tooth from Pradayrol (Caniac-du-Causse, Lot). Stratigraphical and paleontological backgrounds

Marie-Roger Séronie-Vivien et Anne-Marie Tillier

\section{OpenEdition}

Journals

Édition électronique

URL : http://journals.openedition.org/paleo/1523

DOI : $10.4000 /$ paleo. 1523

ISSN : 2101-0420

Éditeur

SAMRA

Édition imprimée

Date de publication : 1 décembre 2002

Pagination : 257-264

ISSN : 1145-3370

Référence électronique

Marie-Roger Séronie-Vivien et Anne-Marie Tillier, «La dent humaine de Pradayrol (Caniac-du-Causse, Lot) dans son contexte stratigraphique et paléontologique », PALEO [En ligne], 14 | 2002, mis en ligne le 17 août 2010, consulté le 27 juillet 2020. URL : http://journals.openedition.org/paleo/1523 ; DOI : https://doi.org/10.4000/paleo.1523

Ce document a été généré automatiquement le 27 juillet 2020

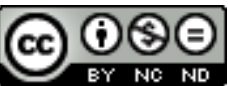

PALEO est mis à disposition selon les termes de la licence Creative Commons Attribution - Pas d'Utilisation Commerciale - Pas de Modification 4.0 International. 


\title{
La dent humaine de Pradayrol (Caniac-du-Causse, Lot) dans son contexte stratigraphique et paléontologique
}

\author{
The human tooth from Pradayrol (Caniac-du-Causse, Lot). Stratigraphical and \\ paleontological backgrounds
}

Marie-Roger Séronie-Vivien et Anne-Marie Tillier

Nous tenons à remercier tous ceux qui nous ont aidé dans ces recherches, plus particulièrement M. Y.Quinif pour sa généreuse coopération, Mlle A.Langlois, MM. J.Dufau, J.L.Guadelli, C.Guérin et J.C.Marquet pour leurs contributions aux déterminations paléontologiques. B. Maureille et M. Seurin sont les auteurs de la figure 3.

1 Le gisement de la grotte de Pradayrol (fig.1), à Caniac-du-Causse (Lot), se présente comme un vaste abri sous roche de plus de 25 mètres de façade et s'étendant en profondeur sur près de 10 mètres. Des départs de couloirs plus profonds sont présents. La puissance du remplissage, qui en certains endroits rejoint la voûte, a été estimé entre 10 et 15 mètres, valeurs confirmées par l'interprétation de profils géophysiques (Ground Penetrating Radar - GPR) enregistrés récemment.

2 L'intérêt archéologique du site a été reconnu en 1996 et une autorisation de sondage fut accordée du 01/04/1998 au 31/12/1998. Elle a permis de réaliser un sondage de quatre mètres carrés poussé jusqu'à un maximum de $90 \mathrm{~cm}$ de profondeur. Les dimensions de ce sondage avaient été choisies pour pouvoir poursuivre la reconnaissance en toute sécurité jusqu'à une profondeur bien plus grande ${ }^{1}$. 
Fig. 1 - Localisation du site de Pradayrol dans le département du Lot Fig. 1 - Location of the Pradayrol site in the Lot area

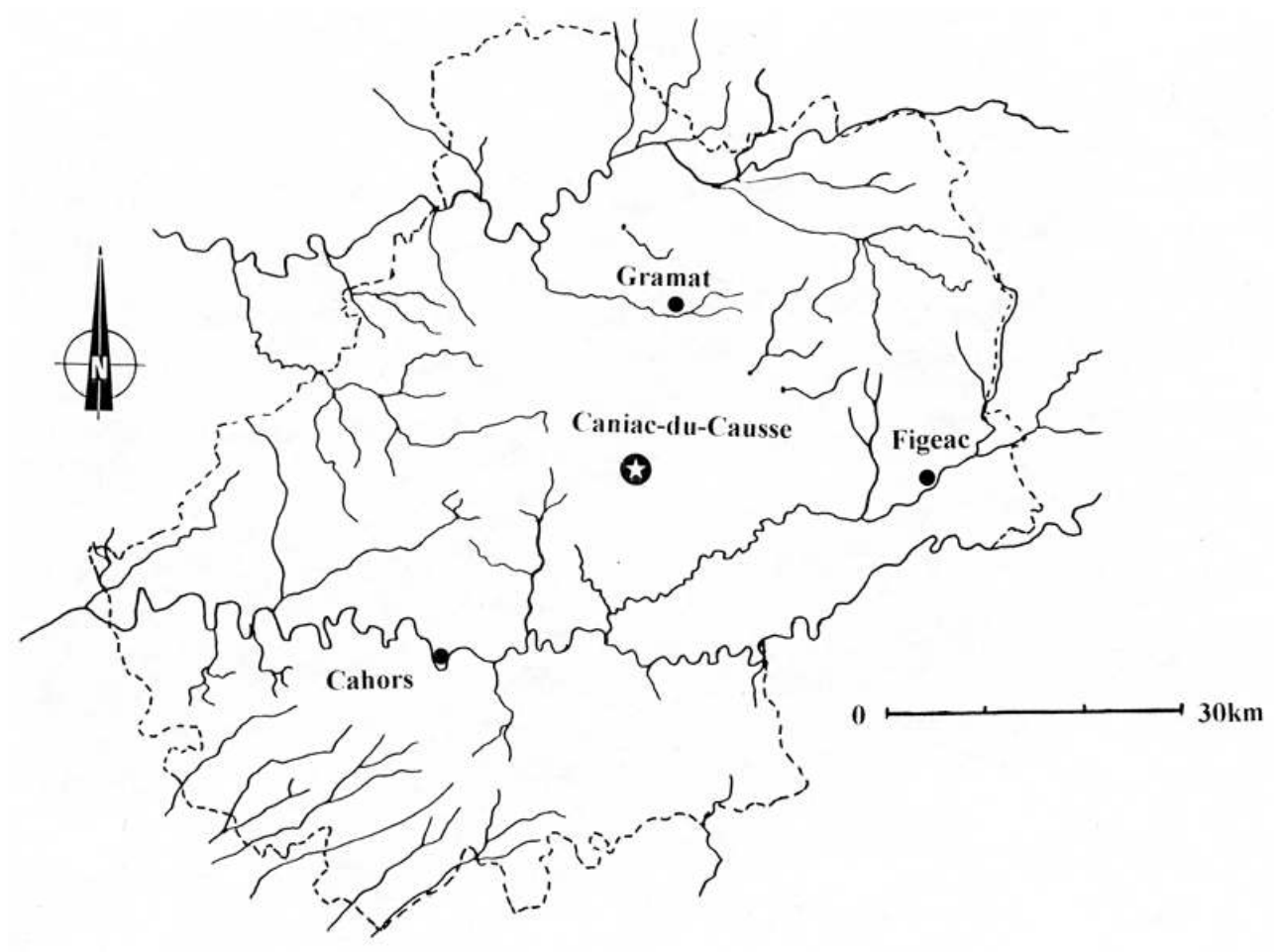

\section{Stratigraphie (fig. 2 )}

Sous une couche très peu épaisse de sédiments meubles, remaniés (couche 1), se développe une unité sédimentaire à dominante argileuse (couche 2) dans laquelle notre reconnaissance a été interrompue. 
Fig. 2 - Coupe stratigraphique de la grotte de Pradayrol et position de la dent humaine (o) Fig. 2 - Stratigraphical section in the Pradayrol cave showing the position of the human tooth (o)

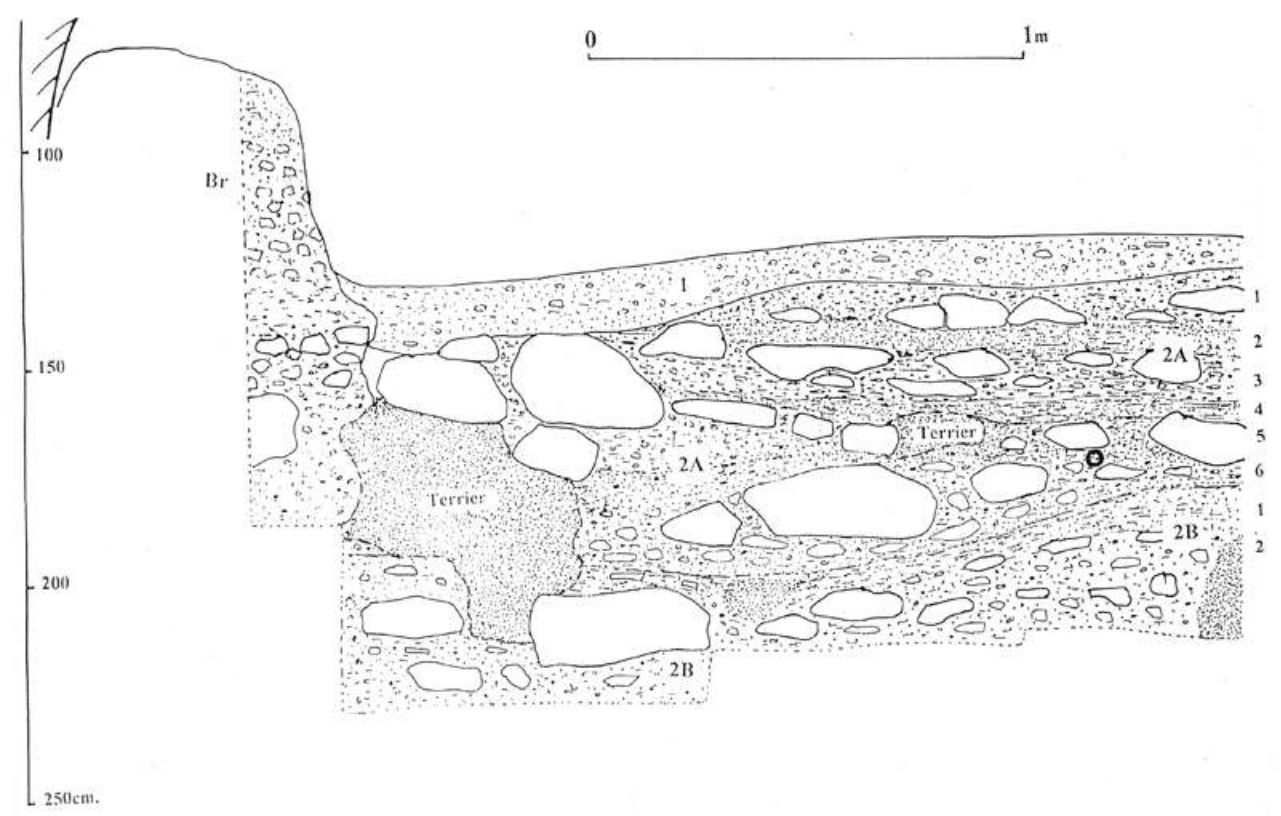

\section{Couche $1:$ (5 à $15 \mathrm{~cm}$ d'épaisseur)}

4 C'est une formation à dominante argileuse contenant des blocs calcaires hétérométriques. Ce dépôt est le produit d'altération de la couche sous-jacente dont elle partage la composition pétrographique. En plus de quelques rares éléments intrusifs récents (os de renards, de blaireaux et de moutons), cette couche a fourni des quartzites taillés et des restes de faune pleistocène (Séronie-Vivien 1999).

\section{Couche 2 : (reconnue sur un maximum de $75 \mathrm{~cm}$ )}

Unité sédimentaire argilo-clastique qui a pu être subdivisée en deux sous-ensembles (couche $2 \mathrm{~A}$ et $2 \mathrm{~B}$ ), séparés par une légère discordance angulaire. Chacun de ces sousensembles peut, à la faveur d'une analyse microstratigraphque fine, se subdiviser en plusieurs séquences dépositionnelles, 6 pour l'ensemble 2A et au moins 2 pour l'ensemble 2B.

La composition de tous ces horizons est caractérisée par une matrice argileuse enrobant des clastes calcaires et des granules de différentes tailles. La matrice argileuse, brun-rouge, est composée non seulement d'argile, mais aussi d'une faible proportion d'éléments détritiques fins, quartz, cristaux isolés de calcite, agrégats noirâtres de nature ferro-manganésifère et quelques paillettes de muscovite. Les granules sont des agrégats arrondis de plusieurs millimètres de diamètre. Ils sont formés d'une partie centrale dans laquelle on peut trouver des débris phosphatés, des fragments osseux, des cristaux de calcite, des éclats de quartzite, etc., et d'une enveloppe (" coating ") calcitique souvent combinée avec des enduits ferromanganésifères. Les clastes calcaires sont des éléments calcaires ou calcitiques, généralement anguleux et hétéromètriques. 
7 L'analyse des structures sédimentaires montre que, au moins pour la partie sommitale, le remplissage provient de l'extérieur de la cavité.

La puissance cumulée de la série étudiée est insuffisante pour étayer une interprétation globale du mode de dépôt et de la nature de l'environnement contemporain. La présence d'éléments évolués phosphatés (granules) enrobant des restes d'origine anthropique (quartzites, os) milite pour l'ancienneté de ce remplissage.

\section{Archéologie}

\section{Industrie}

9 Sur les 1004 objets lithiques recueillis (inventaire provisoire) $87 \%$ étaient en quartzite, $12 \%$ en silex et $1 \%$ de lithologies diverses (quartzites sédimentaires, quartz, andésite).

\section{$1^{\circ}$ ) Quartzites}

10 Ce sont toujours des métaquartzites qui présentent parfois des inclusions de micas et de tourmaline. A l'origine ce furent des galets de rivière qui furent recueillis comme en témoignent les cortex observables. Nous connaissons des lambeaux alluviaux préservés qui contiennent ce type de matériau. Ils se retrouvent dans des fonds de doline à proximité, moins de $800 \mathrm{~m}$ du gisement.

$1161 \%$ des quartzites ont une taille inférieure à $2 \mathrm{~cm}$ ce qui conduit à penser que le site a été un lieu de débitage de ce matériau.

12 Cent dix-huit éléments ont été classés comme outils (13,5\%), parmi lesquels on a distingué $31 \%$ de racloirs, $17 \%$ d'encoches, $22 \%$ de denticulés, $21 \%$ d'outils retouchés, 2 choppers et 2 chopping tools.

13 Cet inventaire conduit à classer cette industrie dans le groupe moustérien (lato sensu), faciès à denticulés. Il faut souligner la très faible représentation du débitage Levallois, une seule pièce, la rareté des pièces bifaciales (1) et des outils sur galet (4).

\section{$\left.2^{\circ}\right)$ Silex}

14 La plupart des silex sont très patinés et il n'est pas possible de déterminer leur nature géologique. Cependant quelques morceaux permettent d'affirmer l'exploitation de sources cénozoïques et sénoniennes.

Les silex sont rares : 120 pièces dont 50 outils. La proportion des outils (42\%) est beaucoup plus importante que pour les quartzites (13,5\%). Par ailleurs, il faut noter que seulement quatre pièces en silex ont plus de $5 \mathrm{~cm}$ de long, tous les autres silex, outils ou éclats, sont plus petits; cela dénote une gestion très parcimonieuse de la matière première.

16 On retrouve la même panoplie d'outils que pour les quartzites avec $23 \%$ de racloirs, $9 \%$ d'encoches, $25 \%$ de denticulés et $25 \%$ d'éclats retouchés. Il est à noter la présence de $8 \%$ d'outils dits "Paléo sup." (grattoirs, perçoirs).

17 Ces résultats, très voisins de ceux obtenus à partir de l'étude des quartzites, renforcent l'hypothèse d'un rattachement de cette industrie au faciès moustérien à denticulés. C'est un faciès déjà connu en Quercy, en particulier aux gisements de La Borde, de 
Coudoulous I, du Mas Vieil, mais dont la position chronologique n'est pas toujours assez précise (Jaubert et al. 1990, Jaubert 1993/1999 et 2001, Niederlander et al. 1956).

\section{Faune}

18 L'inventaire de la faune mammalienne comprend une majorité d'espèces, ou sousespèces, fossiles.

19 Périssodactyles

Dicerorhinus mercki (Jäger, Kaup 1839/41)

Equus hydruntinus Regalia 1904

Equus mosbachensis von Reichenau 1915

20 Artiodactyles

Bos sp. (en cours de détermination)

Cervus elaphus Linné 1758

Cervus simplicidens Guadelli 1994

(autres cervidés en cours de détermination )

21 Carnivores

Hyaena sp.

Canis etruscus Major 1877

Vulpes sp.

Ursus deningeri von Reichenau 1904

22 Lagomorphes

Oryctolagus cuniculus grenalensis Donard 1982

Rongeurs :

Marmota marmota Linné 1758

Hystrix cristata Linné 1758

Microtus agrestis Linné 1758

Microtus arvalis (Pallas 1778)

Microtus brecciensis (Giebel 1847)

Pitymys subterraneus (de Sely-Longchamps 1836)

Chionomys nivalis Martins 1842

Pliomys lenki (Heller 1930)

Arvicola cf. terrestris (Linné 1758)

Apodemus sylvaticus (Linné 1758)

\section{Eléments de datation}

Pour arriver à une estimation de l'ancienneté des niveaux archéologiques, trois démarches étaient possibles :

$\left.1^{\circ}\right)$ Les mesures physiques débouchant sur des valeurs chiffrés, avec des marges d'incertitude plus ou moins grandes. Une première tentative a été faite à partir de niveaux stalagmitiques affleurants dans une partie du site. Malheureusement, les deux analyses réalisées par le Laboratoire de l'université de Mons ( Y.Quinif ) n'ont pas donné de résultats en raison d'une trop grande altération des réseaux cristallins de la 
calcite de ces massifs stalagmitiques. Il reste la possibilité de faire appel à la méthode ESR.

$2^{\circ}$ ) Les informations fournies par la nature de la faune permettent de proposer d'abord une fourchette de dates. La définition du paléo-environnement peut conduire à resserrer cette fourchette. Les espèces fossiles déterminées les plus intéressantes sont :

- Canis etruscus : espèce réputée avoir disparu à la fin de L'Elstérien (Mindel) (stade isotopique 10 : env. -350ka)

- Equus mosbachensis : cheval elstérien ayant disparu pendant le Saalien (stade 7 : env. -200ka)

- Ursus deningeri : autre espèce elstérienne ayant disparu au stade 6 du Saalien (env. -150ka)

- Oryctolagus cuniculus grenalensis : ce lapin est connu des stades 9 à 6 ( de -300 à -150Ka)

D'autres espèces ne fournissent pas des données aussi précises mais peuvent orienter l'interprétation :

- Dicerorhinus mercki : ce rhinocéros est connu jusqu'au stade 3 weischelien, mais il est réputé avoir surtout hanté les steppes de l'Elstérien au Saalien, de -450 à -150ka.

- Equus hydruntinus : cheval connu depuis au moins l'Elstérien où il succède à une forme sténonienne. Il a eu son plein développement du stade 9 au stade 6 (-350 à -150ka), les formes plus récentes sont légèrement différentes anatomiquement (Equus hydruntinus cf. davidi Alimen 1946) (Guadelli 1995).

- Hystrix cristata : si le porc-épic existe encore aujourd'hui, cette espèce thermophile a été signalée seulement dans cinq gisements aquitains pendant les stades 11 à 7 (-450 à 190ka ).

- Lagurus lagurus : ce rongeur migre en fonction de variations environnementales. Il est connu pour avoir fait une incursion dans le sud de la France pendant une partie du Saalien (Marquet 1993 ) entre -300 et -150ka.

Pour tenir compte de l'ensemble de ces données nous retiendrons un intervalle de temps compris entre -150 et -350ka, peut-être même plus étendu en raison de la présence de Canis etruscus.

29 L'approche paléo-écologique propose un environnement steppique, relativement chaud, méditerranéen (porc-épic et campagnol méditerranéen), ce qui conduit à préférer les stades isotopiques $7(-190 / 245 \mathrm{ka})$ du Saalien, $9(-303 / 339 \mathrm{ka})$ de l'Holsteinien, ou même 11 (-362/423 ka) de l'Elstérien.

$\left.303^{\circ}\right)$ La comparaison avec d'autres sites archéologiques nous donne confirmation des conclusions précédentes. Si des industries à dominante de métaquartzites avec prépondérance de denticulés sont connues dans plusieurs gisements quercinois, Mas Viel, Coudoulous I, La Borde, Pailhes, Les Bosses I par exemple, des datations physiques n'ont été obtenues qu' à Coudoulous I et aux Bosses I.

31 A Coudoulous I (Jaubert 1993 à 1999 et 2001), sous un niveau éemien daté de -110 à $-135 \mathrm{Ka}$, il a été reconnu une séquence contenant une faune du Pleistocène Moyen qui a donné une date de -229ka et dont l'âge est estimé entre -200 et -400ka.

Le site Des Bosses I, récemment découvert, a fourni une industrie qu'une description préliminaire permet de rapprocher de celle de Pradayrol. On y a obtenu, par thermoluminescence, des dates comprises entre -250 et -350Ka (Jarry 2001).

En conclusion, et bien que nous ne possédions à ce jour aucune datation directe, il nous semble possible d'avancer pour le niveau de Pradayrol dans lequel on a découvert une dent humaine, une ancienneté supérieure à -150ka, et sans doute voisine de -250 à $350 \mathrm{ka}$. Nous verrions préférentiellement l'occupation de la grotte pendant l'épisode de réchauffement du Saalien (-190/220 ou 245ka) (Broeker 1966), ou pendant 
l'interglaciaire de l'Holsteinien $(-300 / 330 \mathrm{ka})$ ou peut-être une période chaude de l'Elstérien.

\section{Anthropologie}

La dent humaine découverte à Pradayrol est une incisive permanente supérieure dont la racine est cassée à quelques millimètres sous le collet. Sa morphologie générale et sa taille en font une incisive centrale. La surface occlusale présente un degré d'usure élevé et la dentine est exposée sur toute l'étendue du diamètre mésio-distal de la couronne (type 4 de la classification de Molnar 1971).

Cette dent appartient sans conteste à un sujet adulte mais l'âge au décès de ce dernier ne peut cependant être précisé sur la base des éléments dont nous disposons. Une latéralisation de la dent peut être proposée selon deux critères, en dépit de l'usure prononcée de la surface occlusale. Le premier se fonde sur l'observation de la face linguale de la couronne et la reconnaissance d'un bord légèrement plus épais et replié lingualement que l'autre qui correspond au bord distal. Le second critère prend en compte la présence d'une petite surface de contact sur la face mésiale de la couronne indiquant l'usure intersticielle liée au contact de l'incisive centrale adjacente. A l'opposé, sur la face distale de la couronne s'observe un enlèvement d'émail laissant apparaître la dentine. Nous avons donc une incisive droite.

\section{1- Description morphologique}

La couronne de la dent présente une face vestibulaire lisse, avec une convexité de la surface plus marquée dans le sens mésio-distal. Sur la face linguale, en dehors de l'indication d'épaississement des bords latéraux de la couronne, se remarque l'absence de tubercule lingual. Ces éléments ajoutés à l'usure coronaire ne permettent donc pas de se prononcer sur l'existence d'une morphologie en pelle (fig. 3). 
Fig. 3 - Incisive centrale, permanente, supérieure de Pradayrol : faces vestibulaire, linguale et occlusale

Fig. 3 - The Pradayrol upper permanent central incisor: buccal, lingual and occlusal views

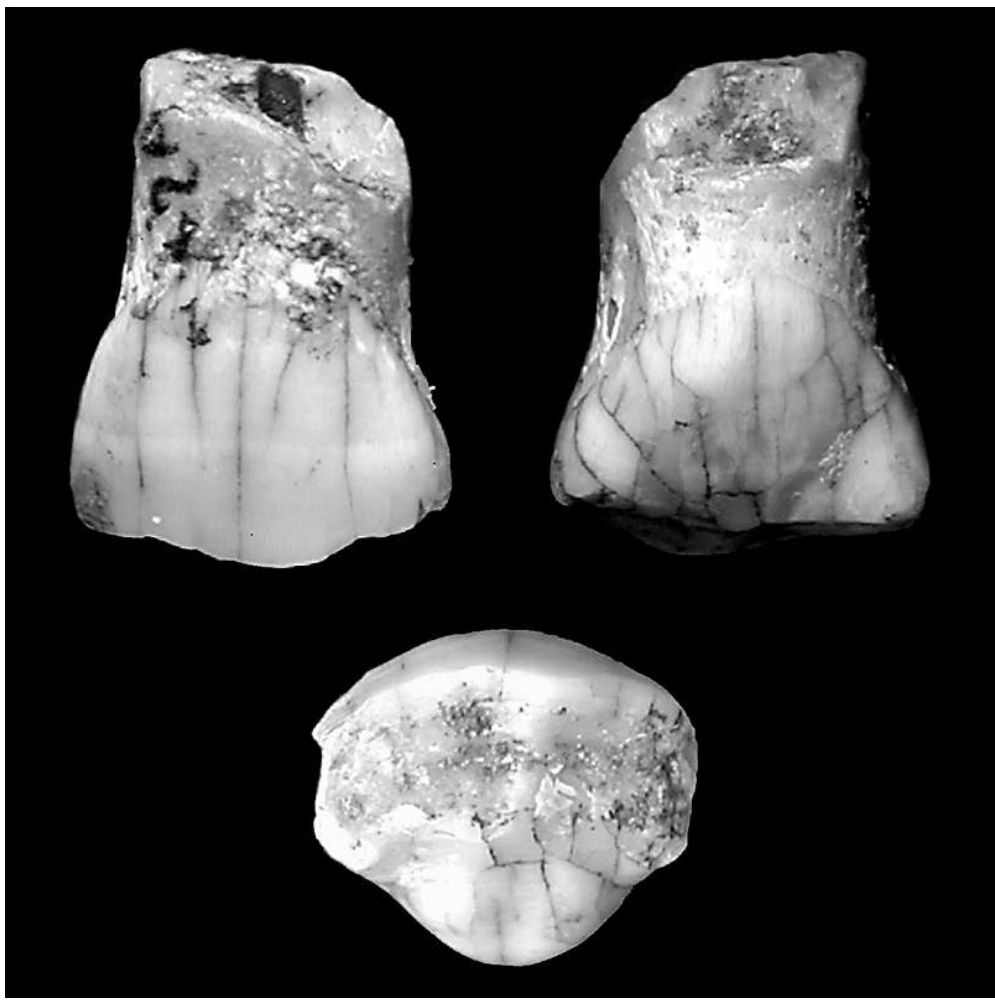

37 La hauteur coronaire ne dépasse pas 7,4 $\mathrm{mm}$ dans son expression maximale côté vestibulaire. Le plan d'usure de la surface occlusale de l'incisive est oblique en direction linguale et légèrement incurvé dans sa partie médiane. Dans la littérature anthropologique, il est souvent fait allusion au degré d'usure avancé des dents antérieures par rapport à celui des prémolaires et molaires comme étant une caractéristique de la denture au Pléistocène moyen et supérieur, dont l'origine est attribuée à des habitudes soit alimentaires soit culturelles.

En dehors de l'importance accordée chez les fossiles à ce caractère qui mérite à elle seule d'être reconsidérée (Zeitoun 1995), remarquons que l'obliquité du plan d'usure sur les dents concernées n'est pas constante dans son expression. Elle peut se faire en direction linguale (e.g. Tabun, Sima de los Huesos, Kebara 27 ; McCown et Keith 1939, Bermudez de Castro 1988, Tillier et al. n.d.) ou en direction vestibulaire (e.g. La Ferrassie 1, Shanidar 1 ; Heim 1976, Trinkaus 1983). Dans le cas des Néanderthaliens sensu stricto l'hypothèse de fonctions paramasticatrices a été avancée par les auteurs, les dents antérieures étant alors perçues comme "une troisième main» (e.g. Smith 1983, Trinkaus 1983). Si occasionnellement une identification de traces d'impacts sur la face vestibulaire des dents fossiles a pu être constatée, cette remarque ne s'applique pas à toutes les dents. Dans le cas de l'incisive de Pradayrol, aucune indication de ce genre ne peut être relevée et l'obliquité linguale du plan d'usure ne favorise guère "l'hypothèse culturelle ». Le type d'attrition observé semble relever plutôt de pratiques alimentaires naturelles. 


\section{2- Analyse métrique}

39 L'incisive de Pradayrol apparaît, par ses dimensions coronaires (diamètre mésio-distal = $9,2 \mathrm{~mm}$; d. vestibulo-lingual $=8,1 \mathrm{~mm}$ ), de grande taille et son développement vestibulo-lingual mérite d'être souligné. La comparaison menée avec les fossiles européens, ceux plus ou moins contemporains représentés par les Néanderthaliens comme les plus anciens, permet de confirmer cette impression, en particulier lorsque sont incluses dans l'échantillon des dents d'enfants.

Les données disponibles pour les fossiles les plus anciens d'Europe Occidentale, proviennent essentiellement de Sima de Los Huesos (Bermudez de Castro 1986) et de La Chaise (Genet Varcin, 1975). Dans le cas des sites de La Chaise, l'usure intersticielle dérivant du contact entre les dents peut expliquer le fait que le diamètre vestibulolingual soit le plus fort. Le site de Krapina en Croatie, quant à lui, rend mieux compte de la variation liée à l'âge des sujets en fonction du nombre d'individus impliqués (Wolpoff 1979).

41 Pour les Néanderthaliens, nous avons volontairement limité la comparaison à deux catégories de données. La première comprend des dents d'enfants pour lesquelles le diamètre mésio-distal correspond à la valeur d'origine car ces dents ne présentent pas d'usure intersticielle. Il s'agit notamment des dents de Chateauneuf 2 et Carihuela pour lesquels les deux diamètres coronaires sont connus (Tillier 1979, Garcia-Sanchez et al. 1994). La seconde source d'informations permet de rendre compte de la variation des dimensions coronaires en fonction de l'âge individuel et elle implique les fossiles de l'Hortus (de Lumley, 1973). C'est ce second type d'informations que nous avons également privilégié lors de l'inclusion, à titre indicatif, d'un échantillon de dents du Paléolithique supérieur, avec la référence au site de Predmosti en Moravie (Matiegka 1934).

42 L'analyse comparative permet de placer la dent de Pradayrol dans la variabilité exprimée par les fossiles européens, qu'il s'agisse des plus anciens, des contemporains ou des plus récents. Ainsi que le montre le tableau 1, il n'apparaît pas de critère métrique pertinent pour proposer une distinction quant à la position de l'incisive de Pradayrol par rapport à la variation des Néanderthaliens de l'Hortus et celle des hommes modernes de Predmosti. En revanche, les incisives de Sima de los Huesos et Krapina, probablement plus anciennes, demeurent plus larges mésio-distalement que celle de Pradayrol.

Tabl. 1 - Dimensions coronaires de l'incisive de Pradayrol comparées à celles de quelques fossiles européens du Pléistocène supérieur. La comparaison tient compte du nombre d'individus Table 1 - Crown dimensions of the Pradayrol incisor compared to those of some european fossils during the Upper Pleistocene, according to individuals number

\begin{tabular}{|l|l|l|}
\hline & Diamètre mésio-distal $(\mathrm{mm})$ & Diamètre vestibulo-lingual $(\mathrm{mm})$ \\
\hline Pradayrol & 9.2 & 8.1 \\
\hline Sima de los Huesos $(\mathrm{N}=4)$ & $9.7 \pm 0.1$ & $7.7 \pm 0.2$ \\
\hline \hline La Chaise Suard 38 & 8.2 & 8.4 \\
\hline
\end{tabular}




\begin{tabular}{|l|l|l|}
\hline La Chaise Bourgeois-Delaunay & 8.9 & 9.9 \\
\hline Krapina (N =11) & $10.3 \pm 0.2$ & $8.9 \pm 0.2$ \\
\hline Chateauneuf 2 & 9.3 & 7.7 \\
\hline Carihuela & 10.6 & 9.0 \\
\hline L'Hortus (N =5) & $8.8 \pm 1.0$ & $7.8 \pm 0.2$ \\
\hline Predmosti $(\mathrm{N}=8)$ & $9.0 \pm 0.3$ & $7.5 \pm 0.3$ \\
\hline
\end{tabular}

\section{3- Pathologie dentaire.}

Sur la face vestibulaire de l'incisive de Pradayrol (fig. 3), à 3,4 mm du collet, s'observe une ligne d'hypoplasie de l'émail qui intéresse toute la largeur de la dent. Cette altération de l'émail correspond au type 4 (LEH) de la classification de la Fédération dentaire Internationale et demeure dans le cas présent peu accentuée.

Selon la charte d'évaluation proposée par Goodman et al. (1980) pour les populations récentes, cette hypoplasie linéaire pourrait correspondre à une altération de la croissance énamélaire se situant autour de 3 ans. Cependant, à propos de cette méthode d'évaluation, des réserves ont été formulées qui se fondent sur la prise en compte de la variation du temps de calcification dentaire au sein des populations actuelles (Berti et Mahaney, 1992). Ajoutons que l'application directe de cette méthode à des dents fossiles sur la base d'une analogie stricte peut, quant à elle, être également discutée.

Quant à l'étiologie de cette pathologie dentaire le consensus n'est pas acquis (e.g. Neiburger 1990, Schultz et al. 1998). La préférence accordée par quelques auteurs aux carences nutritionnelles et donc, par implication, à l'usage parfois abusif des hypoplasies de l'émail comme indicateurs éventuels de trouble de croissance sont à reconsidérer. Dans le cas des fossiles du Pléistocène moyen et supéreur d'Europe, rappelons d'ailleurs que peu de travaux ont été consacrés à cette pathologie dentaire (e.g. Molnar et Molnar 1985, Ogilvie et al. 1989, Brennan 1991, Garcia Sanchez et al. 1994, Bermudez de Castro \& Perez 1995, Skinner 1996) et que leurs résultats et conclusions fluctuent légèrement d'une étude à l'autre. Il s'avère notamment qu'en Europe la fréquence de l'hypoplasie de l'émail est plus faible à Krapina $(26.3 \%$ des dents permanentes, selon Molnar et Molnar) que chez les autres fossiles et notamment les Néanderthaliens plus tardifs ( $35 \%$, selon Ogilvie et al. 1989).

En conclusion, l'incisive de Pradayrol se place dans un intervalle chronologique antérieur au stade isotopique 6 et cette ancienneté fait d'elle, dans l'état actuel des données, un document important. Cette dent humaine ne s'écarte pas de la variation exprimée par les fossiles du Pléistocène moyen et supérieur d'Europe, tant du point de vue morphologique que métrique. A partir de notre connaissance du peuplement humain de l'Europe occidentale, elle peut donc être intégrée à l'ensemble des Prénéanderthaliens, mieux représenté jusqu'alors, pour ce qui est de la France, par les fossiles de Charente, du piémont pyrénéen et la région du pourtour méditerranéen. La 
présence d'une hypoplasie linéaire de l'émail sur la dent de Pradayrol mérite d'être soulignée puisque cette pathologie dentaire est peu connue pour la période considérée.

\section{BIBLIOGRAPHIE}

BERMUDEZ DE CASTRO J.M,. 1986. Dental remains from Atapuerca (Spain), I. Metrics. Journal of Human Evolution, 15, 4: 265 - 288

BERMUDEZ DE CASTRO J.M., 1988. Dental remains from Atapuerca / Ibeas (Spain), II. Morphology. Journal of Human Evolution, 17, 3: 279 - 304.

BERMUDEZ DE CASTRO J.M., PEREZ P.J., 1995. Enamel hypoplasia in the Middle Pleistocene hominids from Atapuerca (Spain). American Journal of Physical Anthropology, 96: 310 - 314

BERTI PR. , MAHANEY M.C., 1992. Quantification of the confidence interval of ninear enamel hypoplasia chronologies. In: LL. Capasso \& AH. Goodman (eds) Recent Contributions to the study of Enamel Developmental defects. J. Paleopath. Monogr. Publ.2, Chieti (Italy): 19 - 30.

BRENNAN M.U., 1991. Health and disease in the Middle and Upper paleolithic of Southwestern France. PhD Dissertation, New York University.

BROECKER W.S., 1966. Absolute dating and the astronomic theory of glaciation. Science,151, $299 / 304$.

GARCIA SANCHEZ M., TILLIER AM., GARRALDA MD. \& VEGA-TOSCANO G., 1994. Les dents d'enfant des niveaux moustériens de la grotte de Carihuela (Grenade, Espagne). Paléo, 6 : 79 - 88.

GENET-VARCIN E., 1975. Etude des dents humaines isolées provenant des grottes de La Chaise de Vouthon (Charente). Bulletins et Mémoires de la Société d'Anthropologie de Paris, 2, Série XIII : 129 145.

GOODMAN A.H., ARMELAGOS G.J., ROSE J.C., 1980. Enamel hypoplasia as indicators in three prehistoric populations from Illinois. Human Biology, 52 : 515 - 528.

GUADELLI J.L., 1995. Etude des équidés. In : M.R.Séronie-Vivien : la grotte de Pégourié, Caniac-duCausse. Préhistoire Quercinoise, Supplément n 5, 100/147.

HEIM J.L.,1976. Les Néandertaliens adultes de La Ferrassie (Dordogne). Etudes anthropologique et comparative. Archives de l'Institut de Paléontologie Humaine, Mém. 35, Paris : Masson.

JARRY M., 2001. Bilan scientifique, année 2000. DRAC Midi-Pyrénées, Service Régional d'Archéologie, Ministère de la Culture et de la Communication, 143/145.

JAUBERT J., 1993/99 et 2000. Bilans scientifiques, années 1992 à 1998 et 2000. DRAC Midi-Pyrénées, Service Régional d'Archéologie, Ministère de la Culture et de la Communication.

JAUBERT J., LORBLANCHET M., LAVILLE H., SLOTT-MOLLER R., TURQ A. \& BRUGAL J-Ph., 1990. Les chasseurs d'aurochs de La Borde : un site du Paléolithique moyen (Livernon, Lot). DAF n²7, 158p.

LUMLEY MA. De, 1973. Anté-néandertaliens et Néandertaliens du Bassin Méditerranéen occidental européen. Etudes Quaternaires, Mém. 2, 626 p., Marseille : Univ. de Provence. 
McCOWN TD., KEITH A., 1939. The Stone Age of Mount Carmel. vol. II, Oxford: Clarendon University Press.

MARQUET J-C., 1993. Paléoenvironnements et chronologie des sites du domaine atlantique français d'âge Pléistocène moyen et supérieur d'après l'étude des Rongeurs. Les cahiers de la Claise. Suppl.N 2, 346 p., Tours.

MATIEGKA J., 1934. Homo Predmostensis. Fosilni clovek z Predmosti na Morave. t. 1: Lebky, t. Ceska Akademie Ved a Umeni , 164 p., Praze. (en tchèque)

MOLNAR S., 1971. Human tooth wear, tooth functiun and cultural variability. American Journal of Physical Anthropology, 34: 175 - 190.

MOLNAR S., MOLNAR I.M., 1985. The Incidence of enamel hypoplasia among the Krapina Neandertals. American Anthropologist, 87 : 536 - 549.

NIEDERLANDER A., LACAM R., CADIERGUES D.R., BORDES F., 1956. Le gisement moustérien du Mas Vieil. L'Anthropologie, LX, 209/235.

NEIBURGER E., 1990. Enamel hypoplasias: poor indications of dietary stress. American Journal of Physical Anthropology, $82: 231-232$.

OGILVIE MD., CURRAN BK.,\& TRINKAUS E., 1989. Incidence and patterning of dental enamel hypoplasia among the Neandertals. American Journal of Physical Anthropology, 79 : $25-41$.

SERONIE-VIVIEN M.R., 1999 . Un nouveau gisement du Paléolithique moyen dans le Lot : La grotte de Pradayrol à Caniac-du-Causse. Préhistoire du Sud-Ouest, 6, 11/12.

SKINNER M., 1996. Developmental Stress in Immature Hominines from Late Pleistocene Eurasia: Evidence from enamel Hypoplasia. Journal of Archaeological Science, 23: 833 - 852.

SMITH F.H., 1983. Behavioral interpretation of changes in craniofacial morphology across the archaic/modern Homo sapiens transition. In E. Trinkaus (ed). The Mousterian Legacy: Human Biocultural Change in the Upper Pleistocene, BAR International series 164: 141 - 163.

SCHULTZ M. , CARLI-THIELE P., SCHMIDT-SCHULTZ T.H., KIEDORF U., KIEDORF H., TEEGEN W-R. \& KREUT K., 1998. Enamel Hypoplasias in Archaeological Skeletal remains. In: KW. Alt, FW. Rösing \& M. Tescher-Nicola (eds). Dental Anthropology. Fundamentals, Limits and Propects. Springer Wien New York, : 293 - 312.

TILLIER A.-M., 1979. La dentition de l'enfant Moustérien Chateauneuf 2 découvert à l'abri de Hauteroche (Charente). L'Anthropologie, 83, 3: 417 - 438.

TRINKAUS E., 1983. The Shanidar Neandertals. New York: Academic Press.

WOLPOFF M.H., 1979. The Krapina Dental remains. American Journal of Physical Anthropology, 50: 67-114.

ZEITOUN V. 1995. Une usure dentaire différentielle chez les Néandertaliens ? Paléo, 7 : 13 -25.

\section{NOTES}

1. Malheureusement, la CIRA à laquelle nous sommes assujettis a considéré, à deux reprises en 1999 et 2001, qu'il était inopportun de continuer cette banale évaluation du patrimoine archéologique régional et qu'il était préférable de prendre des mesures propres à assurer la protection du site, ce qui n'a été l'objet du moindre début de réalisation trois ans après. 


\section{RÉSUMÉS}

Nous présentons dans cette note les premiers résultats d'un sondage réalisé par l'un d'entre nous (M.R.S-V.) dans la grotte de Pradayrol (Caniac-du-Causse, Lot) ainsi que la description d'une dent humaine qui y a été trouvée en 1998. Plusieurs arguments faisant appel à la stratigraphie et à la faune suggèrent que cette dent provient de niveaux antérieurs au stade isotopique 6. L'étude détaillée de la dent, une incisive permanente supérieure, ainsi que la première comparaison avec d'autres fossiles permettent d'inclure cette dent dans la variabilité des premiers représentants de la lignée néanderthalienne. La dent de Pradayrol présente une hypoplasie linéaire de l'émail.

The preliminary results on the excavations conducted by Marie-Roger Séronie-Vivien in the Pradayrol cave (Caniac-du-Causse, Lot) and a description of a human tooth discovered in 1998 are presented in this article. Several arguments based on stratigraphy and fauna support the assignment of the tooth to a time period prior to isotopic stage 6. Detailed morphological description of this tooth, an upper permanent incisor, and comparative analysis with other fossil teeth indicate that the Pradayrol specimen can be included among the early representatives of the Neanderthal population. The Pradayrol tooth represents an early case of linear enamel hypoplasia.

\section{INDEX}

Keywords : Pradayrol, Lot, human tooth, Pleistocene, Neanderthal, enamel linear hypoplasia Mots-clés : Pradayrol, Lot, dent humaine, Pléistocène, Néanderthalien, hypoplasie linéaire de l'émail

\section{AUTEURS}

\section{MARIE-ROGER SÉRONIE-VIVIEN}

Ingénieur géologue, E.N.S.P. - 125, avenue d'Eyzines - 33110 Le Bouscat

\section{ANNE-MARIE TILLIER}

Université de Bordeaux - UMR 5809 du CNRS - Laboratoire d'Anthropologie des Populations du passé - avenue des Facultés - 33405 Talence 